\title{
Composição físico-química do vinho Bordô de Flores da Cunha, RS, elaborado com uvas maturadas em condições de baixa precipitação
}

\author{
Physicochemical composition of Bordô wines from Flores da Cunha, RS, made with grapes matured \\ in low rain conditions
}

Francine Maria Tecchio ${ }^{\mathrm{I}}$ Alberto Miele*II Luiz Antenor Rizzon ${ }^{\mathrm{II}}$

\begin{abstract}
O Bordô é, depois do Isabel, o vinho de mesa de maior importância econômica na Serra Gaúcha, pois existe um considerável segmento de mercado que o aprecia, especialmente por seu sabor frutado e por sua cor intensa $e$ matiz violeta. Devido a isso e às condições de estiagem que ocorreram no verão de 2005, quando as chuvas corresponderam a 38\% da normal climatológica, analisaramse vinhos Bordô do município de Flores da Cunha, RS, um dos mais importantes produtores da Serra Gaúcha. Os vinhos analisados eram varietalmente puros e elaborados segundo a tecnologia de cada vinícola. Avaliaram-se 39 variáveis, das quais os resultados mais expressivos foram os seguintes: álcool $10,58 \% \mathrm{v} / \mathrm{v}$; acidez total $91 \mathrm{meq} \mathrm{L}^{-1}$; acidez volátil $7,3 \mathrm{meq} \mathrm{L}^{-1}$; pH 3,21; extrato seco $24,24 \mathrm{~g} \mathrm{~L}^{-1}$; açúcares redutores $2,90 \mathrm{~g} \mathrm{~L}^{-}$ ; ; extrato seco reduzido $22,34 \mathrm{~g} \mathrm{~L}^{-1}$; cinzas $2,09 \mathrm{~g} \mathrm{~L}^{-1}$; ácido tartárico 5,35g L-1; DO 420 0,480; DO 520 1,296; DO 620 0,184 ; taninos $1,41 \mathrm{~g} \mathrm{~L}^{-1}$; antocianinas $778,8 \mathrm{mg} \mathrm{L}^{-1}$; etanal $14,9 \mathrm{mg} \mathrm{L}^{-1}$; acetato de etila $59,3 \mathrm{mg} \mathrm{L}^{-1}$; metanol $290,9 \mathrm{mg} \mathrm{L}^{-1}$; 1-propanol 24,9mg L ${ }^{-1}$; 2-metil-1-propanol 40,6mg $\mathrm{L}^{-1}$; 2-metil1-butanol 45,9mg L $\mathrm{m}^{-1}$; 3-metil-1-butanol 149,1 $\mathrm{mg} \mathrm{L}^{-1}$; soma dos álcoois superiores $260,5 \mathrm{mg} \mathrm{L}^{-1}$; e K $953 \mathrm{mg} \mathrm{L}^{-1}$.
\end{abstract}

Palavras-chave: Vitivinicultura, Vitis Iabrusca, composição físico-química.

\section{ABSTRACT}

The Bordô, after Isabel, is the table wine presenting the greater economic importance in the Serra Gaúcha region, because there is a segment of the market that likes its characteristics, specially its fruity flavor, color intensity, and violet hue. Due to these aspects and to the very dry climatic conditions in the 2005 summer, where rain represented 38\% of the climatological normal, Bordô wines were analyzed from the commune of Flores da Cunha, RS, Brazil, one of the most important producers of the Serra Gaúcha region. Analyzed wines were varietal ones and made according to the technology of each winery. Thirty nine variables were evaluated and the average parameters of the most expressive ones were the following: alcohol $10.58 \% \mathrm{v} / \mathrm{v}$; total acidity $91 \mathrm{meq}^{-1}$; volatile acidity $7.3 m e q L^{-1}$; pH 3.21; dry extract $24.24 \mathrm{~g} \mathrm{~L}^{-1}$; reducing

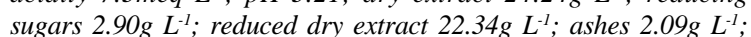
tartaric acid 5.35 $\mathrm{g} \mathrm{L}^{-1}$; A 420 0.480; A 520 1.296; A 620 0.184 ; tannins $1.41 \mathrm{~g} \mathrm{~L}^{-1}$; anthocianins $778.8 \mathrm{mg} \mathrm{L}^{-1}$; ethanal $14.9 \mathrm{mg} \mathrm{L}^{-1}$; ethyl acetate $59.3 \mathrm{mg} \mathrm{L}^{-1}$; methanol $290.9 \mathrm{mg} \mathrm{L}^{-1}$; 1-propanol 24.9mg L-1; 2-mehyl-1-propanol 40.6mg $\mathrm{L}^{-1}$; 2methyl-1-butanol 45.9mg $\mathrm{L}^{-1}$; 3-methyl-1-butanol $149.1 \mathrm{mg} \mathrm{L}^{-1}$; sum of higher alcohols $260.5 \mathrm{mg} \mathrm{L}^{-1}$; and $\mathrm{K} 953 \mathrm{mg} \mathrm{L} \mathrm{L}^{-1}$.

Key words: Vitiviniculture, Vitis Iabrusca, physicochemical composition.

A superfície cultivada com a uva Bordô tem aumentado nos últimos anos, especialmente no município de Flores da Cunha, RS, face à crescente demanda por esta uva. O vinho elaborado com essa uva agrada a um determinado segmento do mercado brasileiro, especialmente devido a sua cor violeta intensa e a seu aroma e sabor frutado e foxado.

A uva Bordô, sendo uma Vitis labrusca, não é cultivada nos países produtores de vinho. Devido a isso, a literatura mundial em relação a essa cultivar e a seus produtos - o suco de uva e o vinho - é praticamente inexistente. Mas no Brasil não há restrição quanto a seu cultivo. Mesmo assim, os trabalhos publicados sobre a uva e o vinho Bordô são restritos (ABRAHÃO et al., 1993; GONÇALVES et al., 1999; ROMBALDI et al., 2004). Face ao exposto, este trabalho foi realizado com o objetivo de caracterizar o vinho Bordô de Flores da Cunha, RS, através de sua composição físico-química.

'Golden Sucos Ltda., Km 5, São Marcos - 1o Distrito, 95180-000, Farroupilha, RS, Brasil. E-mail: golden.s@terra.com.br.

"Embrapa Uva e Vinho, 95700-000, Bento Gonçalves, RS, Brasil. E-mail: miele@cnpuv.embrapa.br. *Autor para correspondência. 
O trabalho foi conduzido em 2005, constatando-se nesse ano precipitação pluviométrica que representou $38 \%$ da normal climatológica da região. Coletaram-se, em junho desse ano, 13 amostras de vinho 100\% Bordô, chaptalizados, em vinícolas do município de Flores da Cunha, RS. As amostras foram analisadas em setembro e outubro, no Laboratório de Enoquímica da Embrapa Uva e Vinho.

Avaliaram-se 39 variáveis relacionadas às análises clássicas, aos compostos fenólicos e aos compostos voláteis e minerais do vinho Bordô. A densidade foi determinada através de um densímetro digital; o álcool, por destilação e densimetria; a acidez total, pela titulação do vinho com $\mathrm{NaOH}$ 0,1N, utilizando o azul de bromotimol como indicador; a acidez volátil, pelo arraste de vapor com titulação do vinho com $\mathrm{NaOH}$ $0,1 \mathrm{~N}$ e fenolftaleína como indicador; e o $\mathrm{pH}$, com um potenciômetro digital, equipado com eletrodo de vidro e calibrado com solução padrão de pH 3,0 e pH 4,0. Os açúcares redutores foram determinados segundo metodologia de MEYER \& LEYGUE-ALBA (1991); o extrato seco e o extrato seco reduzido, segundo RIBÉREAU-GAYON et al. (1982); as cinzas, pela incineração de $20 \mathrm{~mL}$ de vinho em cadinhos de platina a $530^{\circ} \mathrm{C}-550^{\circ} \mathrm{C}$ (AMERINE \& OUGH, 1976); a alcalinidade das cinzas, segundo USSEGLIO-TOMASSET (1995); as antocianinas, segundo RIBÉREAU-GAYON \&
STONESTREET (1965); os taninos, conforme RIBÉREAU-GAYON \& STONESTREET (1966); os polifenóis totais - medindo-se a absorbância a $280 \mathrm{~nm}$ - e os índices de cor - medindo-se a absorbância a 420, 520 e $620 \mathrm{~nm}$-, segundo RIBÉREAU-GAYON et al. (1982); e o ácido tartárico, através da cromatografia líquida de alta performance (AUGUSTE, 1979).

A determinação da maioria dos minerais foi realizada por um espectrofotômetro de absorção atômica Perkin-Elmer equipado com lâmpadas de cátodo oco para cada elemento. Ca, Mg, Mn, Zn, Cu e Fe foram determinados por absorção atômica e K, Na, Li e Rb por emissão de chama (PERKIN-ELMER, 2000). Para a determinação do $\mathrm{N}$ e do $\mathrm{P}$, utilizou-se a metodologia proposta por TEDESCO et al. (1995), adaptada para mostos e vinhos.

A análise dos compostos voláteis foi realizada de acordo com a metodologia de BERTRAND (1975), utilizando um cromatógrafo a gás equipado com detector de ionização de chama. Usou-se o 4-metil-2pentanol como padrão interno. O glicerol também foi determinado por cromatografia gasosa com o mesmo aparelho com que se determinaram os compostos voláteis. Neste caso, o detector foi o de ionização de chama. Utilizou-se o padrão interno 1,6-hexanodiol (CANTAGREL et al., 1978).

Os resultados (Tabela 1) mostram que o vinho Bordô elaborado no município de Flores da

Tabela 1 - Parâmetros analíticos relacionados às análises clássicas e aos compostos fenólicos do vinho Bordô de Flores da Cunha, RS, safra 2005

\begin{tabular}{|c|c|c|c|c|c|}
\hline \multirow{2}{*}{ Variável } & \multicolumn{3}{|c|}{ Parâmetro } & \multirow{2}{*}{ Desvio padrão } & \multirow{2}{*}{$\mathrm{CV}(\%)$} \\
\hline & Maior & Menor & Médio & & \\
\hline \multicolumn{6}{|l|}{ Análises clássicas } \\
\hline Densidade (mg L ${ }^{-1}$ ) & 0,9977 & 0,9948 & 0,9965 & 0,0008 & 0,1 \\
\hline Álcool (\% v/v) & 11,36 & 9,54 & 10,58 & 0,57 & 5,4 \\
\hline Acidez total (meq L $\left.{ }^{-1}\right)$ & 106 & 74 & 91 & 11 & 11,9 \\
\hline Acidez volátil (meq $\mathrm{L}^{-1}$ ) & 11,0 & 5,0 & 7,3 & 1,9 & 26,4 \\
\hline $\mathrm{pH}$ & 3,48 & 3,04 & 3,21 & 0,11 & 3,5 \\
\hline Extrato seco $\left(\mathrm{g} \mathrm{L}^{-1}\right)$ & 30,35 & 18,61 & 24,24 & 3,06 & 12,6 \\
\hline Açúcares redutores $\left(\mathrm{g} \mathrm{L}^{-1}\right)$ & 3,62 & 2,31 & 2,90 & 0,48 & 16,5 \\
\hline Extrato seco reduzido $\left(\mathrm{g} \mathrm{L}^{-1}\right)$ & 28,09 & 17,30 & 22,34 & 2,73 & 12,2 \\
\hline Álcool / Extrato seco & 4,79 & 3,19 & 3,83 & 0,42 & 10,9 \\
\hline Cinzas $\left(\mathrm{g} \mathrm{L}^{-1}\right)$ & 2,70 & 1,25 & 2,09 & 0,40 & 89,1 \\
\hline Alcalinidade das cinzas $\left(\mathrm{g} \mathrm{L}^{-1}\right)$ & 27,0 & 15,0 & 21,3 & 3,4 & 16,1 \\
\hline Ácido tartárico $\left(\mathrm{g} \mathrm{L}^{-1}\right)$ & 6,76 & 4,17 & 5,35 & 0,78 & 14,5 \\
\hline \multicolumn{6}{|l|}{ Compostos fenólicos } \\
\hline DO 420nm & 0,639 & 0,286 & 0,480 & 0,112 & 23,3 \\
\hline DO 520nm & 1,765 & 0,554 & 1,296 & 0,416 & 32,1 \\
\hline DO 620nm & 0,255 & 0,090 & 0,184 & 0,051 & 27,5 \\
\hline Intensidade de cor (DO 420+DO 520) & 2,599 & 0,941 & 1,961 & 0,573 & 29,2 \\
\hline Matiz (DO 420/DO 520) & 0,54 & 0,32 & 0,39 & 0,07 & 19,0 \\
\hline Taninos $\left(\mathrm{g} \mathrm{L}^{-1}\right)$ & 1,95 & 0,89 & 1,41 & 0,33 & 23,6 \\
\hline Antocianinas $\left(\mathrm{mg} \mathrm{L}^{-1}\right)$ & 878,4 & 493,1 & 778,8 & 89,6 & 11,5 \\
\hline Polifenóis totais (I 280) & 104,1 & 48,5 & 79,8 & 13,2 & 16,5 \\
\hline
\end{tabular}

Ciência Rural, v.37, n.5, set-out, 2007. 
Cunha, RS, em 2005, caracterizou-se por apresentar baixo teor alcoólico, apesar das condições climáticas terem sido favoráveis à maturação da uva. Considerese, ainda, que o mosto foi corrigido com sacarose (chaptalização), o que evidencia que essa cultivar possui um baixo potencial de produção de açúcar na região. Quanto às variáveis relacionadas à acidez do vinho, constataram-se elevados valores da acidez total e baixos de $\mathrm{pH}$, o que é uma característica das principais cultivares de Vitis labrusca (RIZZON \& MIELE, 2006). Acidez acima do normal causa desequilíbrio no vinho, prejudicando suas características sensoriais. A acidez constatada foi devida à presença de concentrações relativamente elevadas de ácido tartárico e, também, ao baixo teor da alcalinidade das cinzas.

Características marcantes do vinho Bordô, como a intensidade de cor e o matiz (Tabela 1), são evidenciadas por valores elevados e bem superiores aos dos vinhos tintos da maioria das variedades de uva cultivadas na Serra Gaúcha. Isto pode ser verificado, também, pela concentração de antocianinas desses vinhos. O matiz violeta, por sua vez, que lhe atribui aspecto de vinho jovem, é, em parte, conseqüência da relação DO 420/DO 520, ou seja, da relação existente entre o amarelo dos taninos e o vermelho das antocianinas, e, ainda, da proporção dos cinco grupos de antocianinas predominantes na película da uva Bordô. Por outro lado, o baixo teor de taninos registrados, o que é comum nas variedades americanas, pode ser um dos indicadores da pouca aptidão de amadurecimento e/ou envelhecimento desse tipo de vinho e do tempo de maceração relativamente curto utilizado por ocasião de sua elaboração.

Com relação aos compostos voláteis (Tabela 2), constatou-se que o vinho Bordô tem potencial para produzir metanol, o que pode ser debitado à maior concentração de pectinas presente nas uvas americanas. Por outro lado, observaram-se baixos teores de álcoois superiores, dados que correspondem aos encontrados nos vinhos Isabel elaborados na Serra Gaúcha.

Quanto aos elementos minerais (Tabela 2), constatou-se baixa concentração de potássio, o que explica a presença de elevado teor de ácido tartárico na forma livre e baixo $\mathrm{pH}$ e, conseqüentemente, baixo valor da variável alcalinidade das cinzas.

Portanto, na safra de 2005, o vinho Bordô caracterizou-se por apresentar teor de álcool aquém do esperado, acidez pronunciada, cor intensa, matiz violeta e pouco tanino.

Tabela 2 - Parâmetros analíticos relacionados aos compostos voláteis e aos minerais do vinho Bordô de Flores da Cunha, RS, safra 2005.

\begin{tabular}{|c|c|c|c|c|c|}
\hline \multirow{2}{*}{ Variável } & \multicolumn{3}{|c|}{ Parâmetro } & \multirow{2}{*}{ Desvio padrão } & \multirow{2}{*}{$\mathrm{CV}(\%)$} \\
\hline & Maior & Menor & Médio & & \\
\hline \multicolumn{6}{|l|}{ Compostos voláteis } \\
\hline Etanal $\left(\mathrm{mg} \mathrm{L}^{-1}\right)$ & 25,5 & nd & 14,9 & 7,9 & 53,4 \\
\hline Acetato de etila (mg L $\left.\mathrm{L}^{-1}\right)$ & 83,1 & 43,7 & 59,3 & 10,1 & 17,1 \\
\hline Metanol (mg L $\left.{ }^{-1}\right)$ & 382,5 & 255,0 & 290,9 & 36,5 & 12,5 \\
\hline 1-Propanol $\left(\mathrm{mg} \mathrm{L}^{-1}\right)$ & 36,0 & 16,1 & 24,9 & 6,0 & 24,1 \\
\hline 2-Metil-1-propanol (mg L $\left.{ }^{-1}\right)$ & 55,1 & 29,4 & 40,6 & 9,3 & 22,9 \\
\hline 2-Metil-1-butanol (mg L $\left.{ }^{-1}\right)$ & 50,5 & 34,3 & 45,9 & 4,7 & 10,3 \\
\hline 3-Metil-1-butanol $\left(\mathrm{mg} \mathrm{L}^{-1}\right)$ & 232,2 & 107,4 & 149,1 & 29,3 & 19,7 \\
\hline Soma dos álcoois superiores $\left(\mathrm{mg} \mathrm{L}^{-1}\right)$ & 345,5 & 197,4 & 260,5 & 33,6 & 12,9 \\
\hline \multicolumn{6}{|l|}{ Minerais } \\
\hline $\mathrm{N}\left(\mathrm{mg} \mathrm{L}^{-1}\right)$ & 332,5 & 105,0 & 213,4 & 59,8 & 28,0 \\
\hline $\mathrm{P}\left(\mathrm{mg} \mathrm{L}^{-1}\right)$ & 116,7 & 65,7 & 89,6 & 13,9 & 15,6 \\
\hline $\mathrm{K}\left(\mathrm{mg} \mathrm{L}^{-1}\right)$ & 1.106 & 750 & 953 & 124 & 13,0 \\
\hline Ca $\left(\mathrm{mg} \mathrm{L}^{-1}\right)$ & 109,4 & 73,1 & 94,3 & 13,2 & 14,0 \\
\hline $\operatorname{Mg}\left(\mathrm{mg} \mathrm{L}^{-1}\right)$ & 91,7 & 70,8 & 83,0 & 6,0 & 7,2 \\
\hline $\mathrm{Na}\left(\mathrm{mg} \mathrm{L}^{-1}\right)$ & 22,5 & 2,4 & 10,8 & 5,0 & 46,2 \\
\hline $\operatorname{Mn}\left(\mathrm{mg} \mathrm{L}^{-1}\right)$ & 3,4 & 2,1 & 2,8 & 0,4 & 15,7 \\
\hline $\mathrm{Cu}\left(\mathrm{mg} \mathrm{L}^{-1}\right)$ & 0,9 & 0,1 & 0,3 & 0,2 & 28,3 \\
\hline $\mathrm{Fe}\left(\mathrm{mg} \mathrm{L}^{-1}\right)$ & 3,5 & 1,4 & 2,3 & 0,5 & 23,9 \\
\hline $\mathrm{Zn}\left(\mathrm{mg} \mathrm{L}^{-1}\right)$ & 1,2 & 0,4 & 0,8 & 0,2 & 27,1 \\
\hline $\mathrm{Rb}\left(\mathrm{mg} \mathrm{L}^{-1}\right)$ & 6,6 & 4,2 & 5,0 & 0,7 & 13,0 \\
\hline
\end{tabular}

nd = não detectado.

Ciência Rural, v.37, n.5, set-out, 2007. 


\section{AGRADECIMENTOS}

A autora agradece à Fundação de Amparo à Pesquisa do Estado do Rio Grande do Sul (Fapergs), pela concessão de Bolsa de Iniciação Científica.

\section{REFERÊNCIAS}

ABRAHÃO, E. et al. Folha de Figo: importância e tradição na viticultura de Caldas-M.G. Lavras: Esal, 1993. 40p. (Comunicado Técnico-Científico, 17).

AMERINE, M.A.; OUGH, C.S. Análisis de vinos y mostos Zaragoza: Acribia, 1976. 158p.

AUGUSTE, M.H. Application de la chromatographie en phase liquide à haute pression à l'analyse des moûts et des vins. 1979. 135f. Tese (Doctorat en OenologieAmpélologie) - Institut d'Oenologie, Université de Bordeaux II, Talence.

BERTRAND, A. Recherches sur l'analyse des vins par chromatographie en phase gazeuse. 1975. 291f. Tese (Doctorat d'État ès Sciences) - Institut d'Oenologie, Université de Bordeaux II, Talence.

CANTAGREL, R. et al. Dosage du glycérol dans les vins par chromatographie en phase gazeuse. Revue Française d'Oenologie, Lattes, n.72, p.37-39, 1978.

GONÇALVES, C.A.A. et al. Comportamento da cultivar Folha de Figo (Vitis Iabrusca L.) sobre diferentes porta-enxertos de videira. Revista Brasileira de Fruticultura, Jaboticabal, v.24, n.4, p.7-14, 1999.
MEYER, C.R.; LEYGUE-ALBA, N.M.R. Manual de métodos analíticos enológicos. Caxias do Sul: UCS, 1991. 51p.

PERKIN-ELMER. Analytical methods for atomic absorption espectrophotometry. Singapura: Perkin-Elmer, 2000. 300p

RIBÉREAU-GAYON, J. et al. Traité d'oenologie: sciences et techniques du vin: analyse et contrôle des vins. 2.éd. Paris: Dunod, 1982. V.1. 645p.

RIBÉREAU-GAYON, P.; STONESTREET, E. Dosage des tanins du vin rouge et détermination de leur structure. Chimie Analytique, Paris, v.48, n.4, p.188-196,1966.

RIBÉREAU-GAYON, P.; STONESTREET, E. Le dosage des anthocianes dans le vin rouge. Bulletin de la Societé Chimique de France, Paris, v.9, n.419, p.2649-2652, 1965.

RIZZON, L.A.; MIELE, A. Efeito da safra vitícola na composição da uva, do mosto e do vinho Isabel da Serra Gaúcha, Brasil. Ciência Rural, Santa Maria, v.36, n.3, p.959-964, 2006.

ROMBALDI, C.V. et al. Produtividade e qualidade de uva, cv. Bordô (Ives), sob dois sistemas de cultivo. Revista Brasileira de Agrociência, Pelotas, v.10, n.4, p.519-521, 2004

TEDESCO, M.J. et al. Análises de solo, plantas e outros materiais. 2.ed. Porto Alegre: Departamento de Solos, Faculdade de Agronomia, UFRGS, 1995. 174p. (Boletim Técnico, 5)

USSEGLIO-TOMASSET, L. Chimica enológica. 4.ed Brescia: AEB, 1995. 343p. 